\title{
On the Harmfulness of Secondary Game Objectives
}

\author{
Erik Andersen, Yun-En Liu, Richard Snider, Roy Szeto, \\ Seth Cooper, and Zoran Popović \\ Center for Game Science \\ Department of Computer Science \& Engineering, University of Washington \\ \{eland,yunliu,sniderrw,szetor,scooper,zoran\}@cs.washington.edu
}

\begin{abstract}
Secondary game objectives, optional challenges that players can choose to pursue or ignore, are a fundamental element of game design. Still, little is known about how secondary objectives affect player behavior. It is commonly believed that secondary objectives such as coins or collectible items can increase a game's flexibility, replayability, and depth. In contrast, we present results from analysis of two popular online Flash games showing that secondary objectives can easily harm the retention of many players. We support our findings with data collected from over 27,000 players through large-scale A/B tests in which we measured play time, progress, and return rate. We show that while secondary objectives can encourage long-term players to extend their playtime, they can also cause many players to play for less time. By modifying secondary objectives so that they reinforce the primary goal of the game instead of distracting from it, we are able to avoid negative consequences and still maintain the retention of long-term players. Our results suggest that secondary objectives that support the primary goal of the game are consistently useful, while secondary objectives that do not support the main goal require extensive testing to avoid negative consequences.
\end{abstract}

\section{Categories and Subject Descriptors}

K.8.0 [Personal Computing]: General - Games

\section{Keywords}

games, secondary objectives, analytics, A/B testing

\section{INTRODUCTION}

Modern games are filled with secondary objectives: optional challenges that reward the player upon completion or simply exist for their own sake. Game designers add these objectives for several reasons: to give freedom and flexibility to the player, to give the player something to do in between difficult segments of the game, and to provide a way for expert players to distinguish themselves. It is widely believed

Permission to make digital or hard copies of all or part of this work for personal or classroom use is granted without fee provided that copies are not made or distributed for profit or commercial advantage and that copies bear this notice and the full citation on the first page. To copy otherwise, to republish, to post on servers or to redistribute to lists, requires prior specific permission and/or a fee. that secondary objectives can add entertainment value to a game because players can choose to attempt them or simply ignore them. However, their exact effect on player engagement and retention remains unknown. While secondary objectives can motivate players, they can also distract players from the primary objectives of the game, possibly with negative consequences.

The popularity of online casual game websites and the ease with which new versions of a game can be uploaded provides an ideal testbed for examining the effects of game design decisions such as these. Kongregate, one of the most popular websites for free online games, attracts 10 million players who collectively spend 23 million hours playing games per month [1]. A recent examination of two online Flash games from different genres that we developed and released to Kongregate, Refraction and Hello Worlds, showed that the presence of optional challenges caused the median player to play significantly less [2]. These surprising results motivated us to explore secondary objectives in further depth. Therefore, we conducted a new series of large-scale distributed A/B tests in which players received variations of secondary objectives. We measured the effects of these variations on how much progress players made in each game, how long they played, and how likely they were to return.

We provide quantitative evidence that players respond differently to optional challenges; these challenges seem to motivate expert players while causing other players to give up sooner. In one game, the group of players who quit earlier due to secondary objectives was four times as large as the group who played longer. We also show that secondary objectives that reinforce the primary objectives, rather than distracting from them, can improve the long-term retention of experts while not losing other players. Our results suggest that game designers should design secondary objectives carefully, and should not assume that players will ignore them if they find them frustrating. Furthermore, as these effects may be hard to find without large amounts of data, they point to the importance of gathering and analyzing this data from a game's target population. Further research is necessary to know whether our results generalize to other games and genres. Our methodology offers a promising way to investigate the impact of different aspects of games on player behavior, and may help researchers develop a comprehensive and empirical model of player motivation in games. 


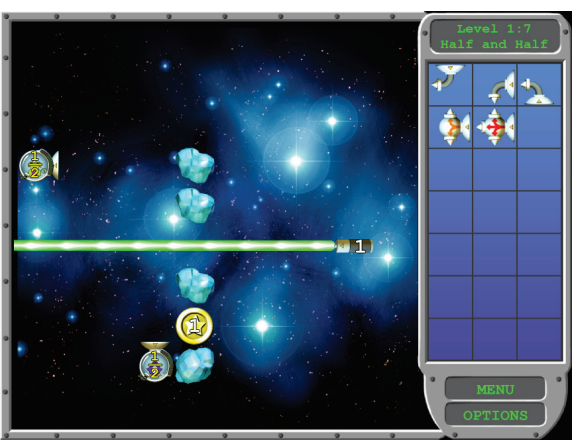

(a)

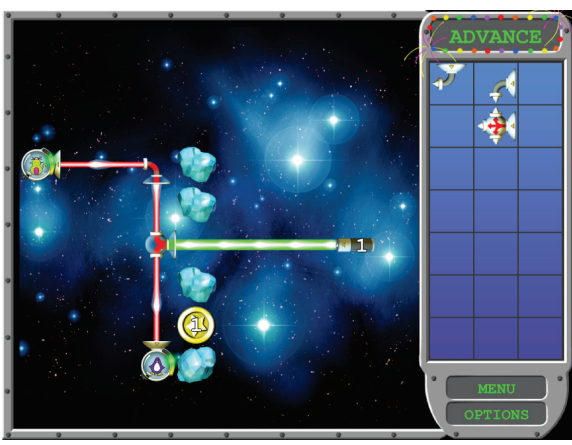

(b)

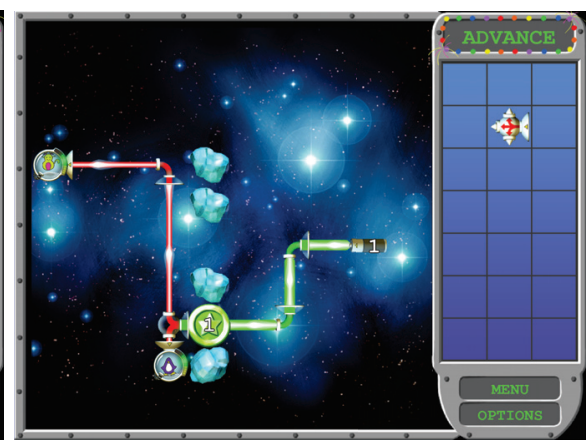

(c)

Figure 1: A level of Refraction. The goal is to use the pieces on the right to split lasers into fractional pieces and redirect them to satisfy target spaceships. 1(a) shows a level that requires the player to direct $1 / 2$ to two spaceships. There is also an optional coin that requires 1. 1(b) is the most natural solution to this level, only using two pieces. $\mathbf{1 ( c )}$ is the more advanced solution that also satisfies the coin as well as the targets. We tested the effects of these optional challenges on player behavior.

\section{RELATED WORK}

Many guidelines and recommendations exist for how to design objectives in games. Schell states that a game's goals should be concrete, achievable, and rewarding [19]. Secondary objectives add more potential goals to a game, and thus should still satisfy these criteria. James Gee [7] has argued that players enjoy games most when they are learning, and that good learning in games occurs when problems are well-ordered and pleasantly frustrating. Adding secondary objectives may affect the real or perceived challenge a game offers, changing these properties in non-obvious ways. Gazzard [6] discusses the potential impacts of rewards that do not have significant gameplay impact but are still perceived as rewards by players. These rewards can be used to teach the player and show them they are on the right path; however, they can also allow the creation of player-defined goals that differ from the main designed goals in the game.

A particularly relevant concept is Csikszentmihalyi's theory of flow [4]. According to this theory, players reach maximal enjoyment when a game's challenge is matched to their skill level. If a game presents a secondary objective to a user, and the skill required to accomplish that objective is far above the player's current skill level, then accomplishing this objective could push the player into the region of anxiety. Our work statistically analyzes how the design of such secondary objectives can affect a game.

A common approach to deriving rules for game design is to distill principles by observing games. Hullet and Whitehead [11] studied design patterns in FPS levels, with the goal of understanding cause-effect relationships and leading to more interesting level design. Milam and Nasr [15] developed a framework for 3D level design to uncover how designers can "push and pull" players through a level. Smith et al. [21] analyzed 2D platformer levels to develop a hierarchical model of the components that make up levels. Our work differs by focusing on secondary objectives within games and testing design decisions with data gathered from a large number of players.
Players have many reasons to be motivated to play games. Motivation theory considers activities such as playing games and solving puzzles to be intrinsically rewarding [5] for many, but not all people. Intrinsic motivation can be enhanced when the needs of competence, autonomy, and relatedness are met [18] and undermined when they are not. Divergent goals that are difficult to complete may undermine players' feelings of competence and diminish motivation. Harackiewicz and Elliot [10] showed that depending on the achievement orientation of an individual, either performance or mastery goals can enhance intrinsic motivation in the context of pinball games. Ryan et al. [17] demonstrated that feelings of competence are associated with preference for continued play of games. A taxonomy of the types of players and their motivations and goals in online games was proposed by Bartle [3]. Following that, Nick Yee [22] surveyed thousands of MMORPG players, finding that there are many factors that motivated players in these games.

Frustration may also have an impact on player response to games. Some authors have examined the effects of frustration on task performance. In the context of the World Wide Web, Selvidge et al. [20] showed that delays in the loading of websites can lead to increased frustration and decreased task completion. The delayed gratification of difficult objectives in browser games may lead to frustration and quitting among casual users. Further, Klein et al. [12] showed that acknowledgment of player frustration while playing a game (induced in the form of random delays while playing) may have an effect on how long players will freely play the game. We believe secondary objectives have the potential to frustrate players if not designed correctly.

The vast quantities of data made available by the Internet have enabled many data-driven analyses for various purposes. Recently, Lewis and Whitehead [14] collected a large amount of data about World of Warcraft players from the World of Warcraft Armory, a publicly-available database of player behavior. Others use the ability to dynamically change content to run large-scale experiments. Amazon has used this approach, in conjunction with various metrics, to test different website layouts seeking to maximize sales [13]. 


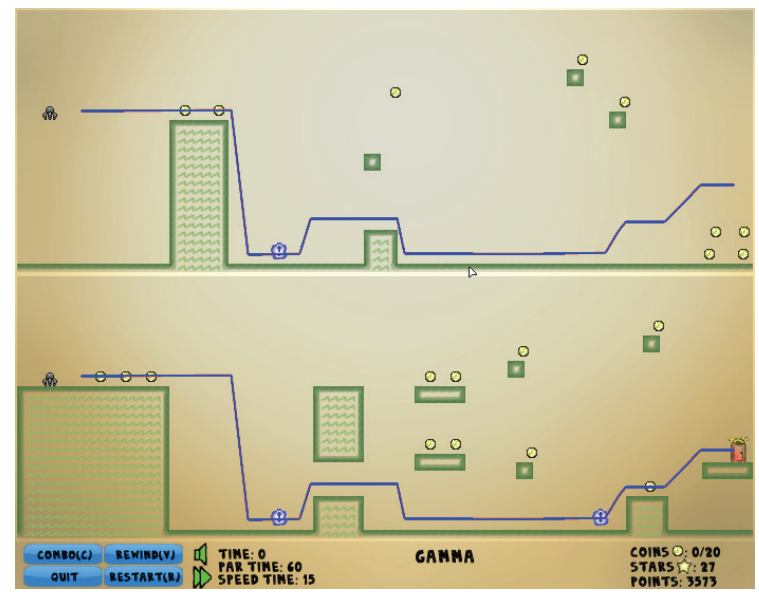

Figure 2: A level of Hello Worlds. Some of the coins are on the main path (in blue) and reinforce the primary goal of completing the level. Others are off the path and difficult to obtain. In this work, we study what effects these different types of coins have on player retention.

Google Website Optimizer [8] makes this basic approach available to webmasters for their sites. A similar approach has been applied to games. Zynga relies on metric-driven design based on player behavior to tune their games; for example, they discovered that pink fonts caused players to click on an advertisement for PetVille far more often [9].

\section{METHODOLOGY}

In our experiments, we examined two Flash games that we developed. The first, Refraction, is shown in Figure 1. Refraction was created by a team of graduate and undergraduate students as part of a larger games for learning project. Refraction was released in September of 2010 and has been played over 300,000 times on Kongregate. Each level of the game is played on a grid, and consists of laser sources, target spaceships, and asteroids that all cannot be moved. Each spaceship desires a target fraction of the laser, indicated by the yellow number on each spaceship. The player can satisfy the targets by manipulating pieces that change the direction of the laser and splitters that split the laser equally into two or three parts. The player must satisfy all of the spaceships at the same time with the correct amounts to complete the level, as shown in Figure 1(b).

Some levels of Refraction also include coins as secondary objectives, such as the level in Figure 1(a). Each coin has a desired target amount, similar to the target spaceships, and transmits lasers instead of absorbing them. If the player manages to send the correct amount through the coin and satisfy all of the level's target spaceships, as shown in Figure $1(\mathrm{c})$, the player will "get" the coin upon completion of the level. The coins are all designed so that the configuration of lasers that satisfies the coin is more difficult and less intuitive to find than the simplest solution.

The second game we studied is Hello Worlds, a puzzleplatformer game shown in Figure 2. Hello Worlds was created by a team of undergraduate students for a video game design capstone class and has been played over 1,000,000 times on Kongregate since its release in May 2010. In Hello Worlds, the player exists in multiple worlds simultaneously, interacting with all of the worlds at the same time. If one character hits a wall, they all hit a wall.

Similar to many platformer games such as Sonic the Hedgehog and Super Mario Brothers [16], most levels of Hello Worlds feature coins scattered throughout the level. The player can collect these coins to achieve a higher score, and they also unlock levels later in the game. The level shown in Figure 2 has a typical number of coins. In general, coins are scattered throughout the levels, with some on the main path through a level, and some far away from the path. The coins off of the path are harder to collect.

To collect data on a large scale, we used A/B testing and player metrics. In A/B testing, researchers present multiple conditions randomly to users in the same population and see how they respond, allowing them to evaluate system components quantitatively. In our experiments, the game randomly assigned new players to one of the experimental conditions when they loaded the game. We focused solely on new players; veteran players who had already played the game were not included in our analysis. We tracked players using the Flash cache, which is preserved over time and is commonly used by Flash games to store player progress. It is separate from the browser cache. If the player returned to play the game at a later date, the saved variables ensured that the player received the same experimental version of the game. There was always a chance that returning players might be treated as a new player if they cleared their Flash cache, used another browser, or used another computer. However, we judged this risk to be minimal.

We gathered data by instrumenting our games to record player events and send them to our server. Both games reported their own unique set of events. We measured player engagement with three metrics. First, we counted the total number of unique levels that each player completed. Second, we measured the total amount of time that each player spent playing the game. Since players occasionally idle for long periods while playing Flash games, we aggregated moves the player made in 30-second intervals. If two or more consecutive intervals (a full minute) had no player actions, this time was discounted from the total play time. Finally, the third measure of engagement that we used was return rate. If a player loaded the game again sometime after his or her first play session, we counted that player as a returning player. If the player returned, we added any new levels completed or time played to that player's total.

The period of data collection was slightly different for each game. We collected data from Hello Worlds for approximately four weeks. During this time, Hello Worlds received 3,000 new players whom we included in this experiment. As for Refraction, Kongregate agreed to place the game on the front page for approximately four days. Being on the front page significantly increased the rate of new players; we report data from approximately 24,000 players who played during this time period. 
Collecting data anonymously through online portals has strengths and weaknesses. A key advantage is that, in contrast to in-house playtesting, our experiments are conducted "in the wild" where players do not know they are part of an experiment. As a result, players are playing under their own motivation, and our findings are based solely on their observable behavior. However, a key limitation is that we have no interaction with the participants, and we cannot know what they are thinking or feeling. Therefore, we must infer their level of engagement from their behavior. We believe that our metrics of progress, time played, and return rate are reasonable measurements of engagement and that most game designers wish to maximize these attributes. Since a new free online Flash game is always just a few clicks away on Kongregate, we believe that players will quit if they become too bored or too frustrated.

\section{RESULTS}

We created three different versions of each game in order to examine secondary objectives. As a baseline condition, we used a version of each game with no coins at all. In this version, we also removed components of the interface that would suggest the presence of coins, such as the "Trophy Room" in Refraction and the coin counter on the status bar in Hello Worlds. The other two conditions tested specific theories about coins that will be described in the following sections. Other than the variations in secondary objectives, the different versions of the game were exactly the same.

Our measurements of levels completed and time played were not normally distributed, and we therefore relied on a nonparametric test, the Wilcoxon / Kruskal-Wallis 2-sample test, to analyze levels completed and time played for largescale effects. For return rate, we used Pearson $\chi^{2}$ analyses to compare the percentages. The the variance in our measures was very high, so a lot of data was necessary in order to show statistical significance at the $\mathrm{p}=0.05$ level. Since we were only able to attract about 1000 people for each condition in Hello Worlds, we did not collect enough data for some comparisons to be statistically significant. However, since the trends for Hello Worlds match what we observed in [2], we believe that these comparisons would be significant if we had collected more data.

\subsection{Off-path Coins}

We hypothesized from the results in [2] that the difficulty of the coins in both games might be causing frustration and harming player engagement. Therefore, we created a version of each game in which the coins are unrelated to the primary goals of each level, which we call "off-path coins". The coins in Refraction are all divergent from the main solution to each level, so the off-path coins version of Refraction was just the normal game. However, in Hello Worlds, the coins are sometimes located on the primary path through the level and sometimes located off of it, as shown in Figure 2. Therefore, we created an off-path coins version of Hello Worlds where all of the coins are off of the main path.

We first examined the effect of secondary objectives on completion of primary objectives by comparing the versions with no coins and off-path coins. 7844 players played Refraction without coins and 8034 players played with off-path coins. Players completed significantly more levels in the version

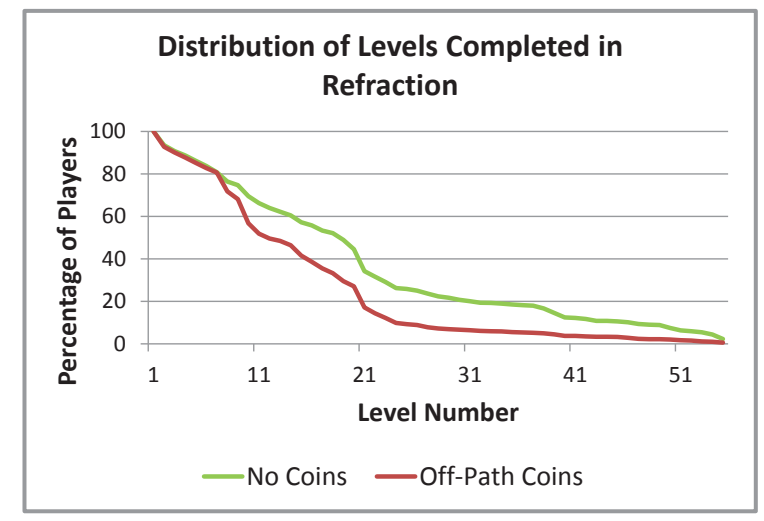

Figure 3: Graph of levels completed for Refraction, comparing the versions of the game with off-path coins and no coins. The $\mathrm{x}$-axis shows level numbers, and the y-axis shows the percentage of players who complete that level. The game is linear; players cannot attempt a level without completing the one before it. Players complete dramatically fewer levels in the version with off-path coins, most likely because they are spending their time trying to get the coins, and not advancing in the game. These players quit earlier and never return to complete the later levels in the game. The percentage of people who complete the game is approximately the same in both conditions, around $5 \%$.

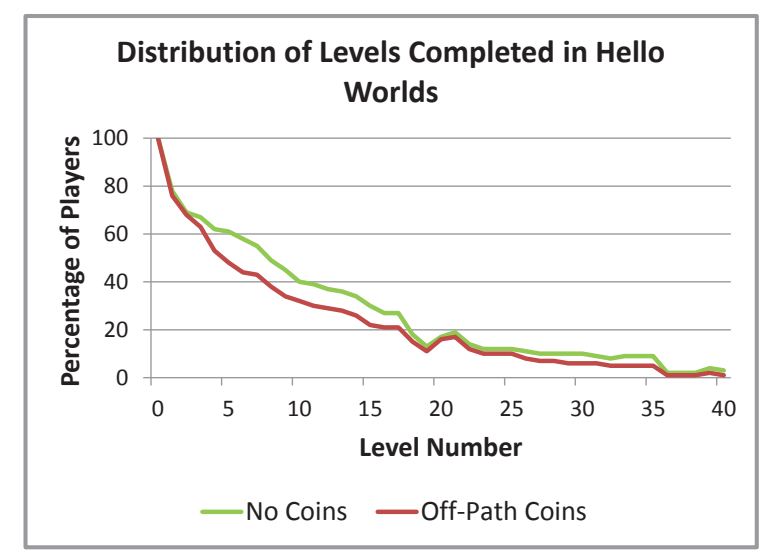

Figure 4: Graph of levels completed for Hello Worlds, comparing the versions of the game with off-path coins and without coins. The game is somewhat nonlinear and players can complete some levels out of order. Like in Refraction, players complete fewer levels in the version with off-path coins. This is further evidence of how secondary objectives can distract the player from the primary objectives of the game. 


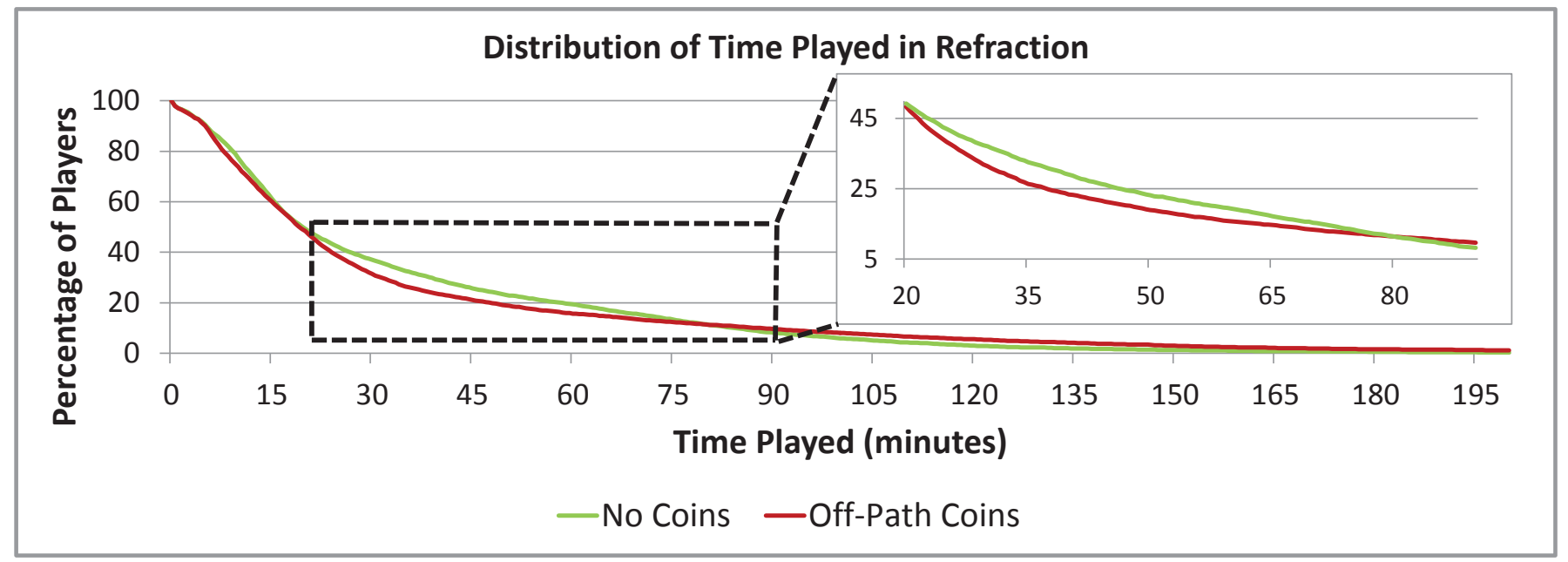

Figure 5: Graph of time played for Refraction, comparing the versions of the game with off-path coins and with no coins. The $\mathrm{x}$-axis shows lengths of time since starting the game, and the $\mathrm{y}$-axis shows the percentage of the players who play for at least that much time. The tail of this graph shows that off-path coins do cause some players to play longer. We think that this may be the expert players who are trying to complete everything in the game, and for whom coins may be a big motivating factor. Off-path coins do not seem to increase play time in Refraction, however, until after 80 minutes, when $90 \%$ of the players have quit. Before this, especially from about 15 minutes to 80 minutes, players quit more in the version of the game with off-path coins.

with no coins (Median $=20$ levels) than with off-path coins $(\mathrm{M}=17), \mathrm{Z}=24.788, \mathrm{p}<0.0001$. This represents an increase of $17.6 \%$ for the median player. Players played significantly more in the version with no coins ( $\mathrm{M}=1170$ seconds) than the version with off-path coins $(\mathrm{M}=1140), \mathrm{Z}=4.696$, $\mathrm{p}<0.0001$. The difference in return rate was not significant. $19.48 \%$ of players without coins returned, while $19.24 \%$ of players with off-path coins returned, $\chi^{2}=0.566, \mathrm{p}=0.452$.

1003 players played Hello Worlds with no coins and 947 played with off-path coins. Players completed significantly more levels with no coins $(\mathrm{M}=7)$ than with off-path coins $(\mathrm{M}=4), \mathrm{Z}=-3.810, \mathrm{p}<0.0001$. The median player played longer without coins $(\mathrm{M}=480)$ than with off-path coins ( $\mathrm{M}$ $=360$ ), although this effect was not statistically significant, $\mathrm{Z}=-0.688, \mathrm{p}=0.492$. The change in the return rate was also not significant, decreasing from $20.44 \%$ without coins to $18.37 \%$ with off-path coins, $\chi^{2}=1.328, p=0.249$.

These statistics show that off-path coins cause players to complete fewer levels and play for less time. This difference in levels completed is shown for Refraction in Figure 3, and for Hello Worlds in Figure 4. In both games we see that players stop playing much earlier in the game when there are coins. These graphs show how secondary objectives can distract players from the primary goals of the game.

We also examined how the presence of secondary objectives influenced play time in both games. Figure 5 points to an interesting effect in Refraction: off-path coins cause some players to play longer and others to play less than players without coins. The distribution can be divided into three different regions. The $50 \%$ of players who quit within the first 15 minutes behave similarly, although people quit slightly sooner in the version with coins. The next $40 \%$ of peo- ple, who quit between 15 minutes and 80 minutes, quit as much as 10 minutes earlier than they would have otherwise. Finally, the $10 \%$ of players who quit last play longer with coins. The distribution of time played for Hello Worlds, as seen in Figure 6, shows the same effect. The $40 \%$ of players who quit first behave similarly between the two conditions, but the next $30 \%$ quit earlier in the version with coins. The coins motivate the $30 \%$ who quit last to play longer.

In both games, these results show that secondary objectives benefit some players while harming the retention of others. Players who already play for a long time play even more when coins are present. However, a large set of players who play the games for a moderate amount of time actually play less when off-path coins are present. While it is challenging to quantify the number of players whom we "lose" due to off-path coins, the insets of Figures 5 and 6 show that the gap between these two conditions widens to as much as $6 \%$ in Refraction and $4 \%$ in Hello Worlds. This means that when the gap is at its widest, the off-path coins versions are missing $19 \%$ of the Refraction players and $8 \%$ of the Hello Worlds players who would otherwise still be playing if there were no coins. However, this gap eventually closes and reverses as play time increases. As a result, the off-path coins in both games effectively bias the game to engage longterm players at the expense of intermediate players.

A surprisingly large number of players quit earlier with offpath coins. For Refraction, the population that is negatively impacted by off-path coins is roughly four times as large than the positively impacted population. In Hello Worlds, the negatively and positively impacted populations are about the same size. This trade-off may be acceptable, but that depends on the designer's goals for the game. 


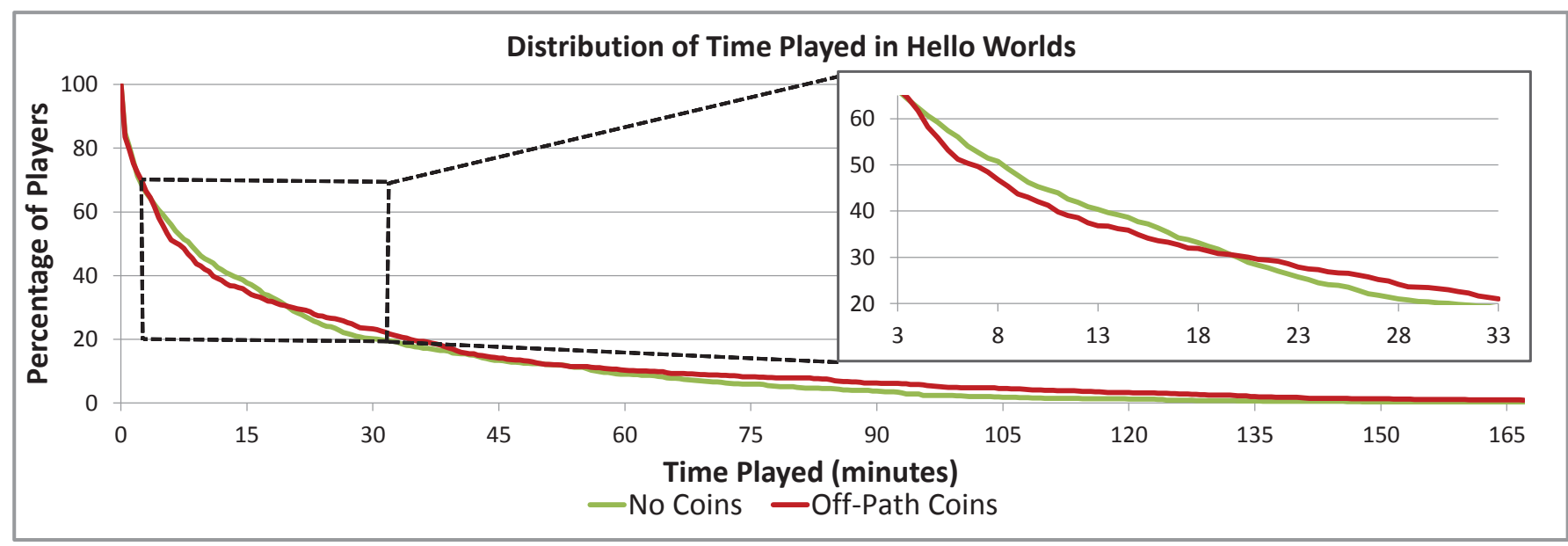

Figure 6: Graph of time played for Hello Worlds, comparing the versions of the game with off-path coins and with no coins. The x-axis shows lengths of play time, and the $y$-axis shows the percentage of the players who play for at least that much time. As in the case of Refraction, the tail of this graph shows that off-path coins increase the play time of the $30 \%$ of players who play the longest. These are the players who play more than 18 minutes. However, the $30 \%$ of players who quit between 3 minutes and 18 minutes into the game play less when off-path coins are present. This provides further evidence that secondary objectives can be a double-edged sword because players are incentivized differently.

\subsection{On-path Coins}

We hypothesized that the coins in both games may be particularly distracting and frustrating because they are unrelated to the primary objectives of each level. Thus, solving the coins does not help the player solve the level, and in some cases may even obscure the most logical path by leading players astray. We observed that in some highly successful games such as Super Mario Brothers [16], the coins are not far off of the main path through the level. Therefore, we tested putting the coins onto the main path of each level so that they supported the primary objectives of the game. In Hello Worlds, this meant putting the coins onto the easiest way through each of the levels. To put the coins "on the path" in Refraction, we moved them onto what we felt was the most natural solution to each level. This change not only decreased the amount of effort necessary to acquire each coin, but also gave the user a hint about how to solve each level.

Player engagement improved considerably. The 7878 players with on-path coins in Refraction played significantly more levels than players with off-path coins, increasing the median from 17 to $20, \mathrm{Z}=17.538, \mathrm{p}<0.0001$. Time played increased as well, from a median of 1140 seconds to $1230 \mathrm{sec}-$ onds, $\mathrm{Z}=6.578, \mathrm{p}<0.0001$. The return rate also increased from $19.01 \%$ to $20.98 \%, \chi^{2}=9.658, p=0.002$. Comparing on-path coins with no coins, the median players both played 20 levels, $\mathrm{Z}=6.274, \mathrm{p}<0.0001$. We found a significant effect for time played, with players with on-path coins (M $=1230)$ playing longer than players with no coins $(\mathrm{M}=$ $1170), \mathrm{Z}=-2.030, \mathrm{p}=0.02$. The return rate also increased; $20.98 \%$ of people returned with on-path coins, compared to $19.48 \%$ of players with no coins, $\chi^{2}=5.479, \mathrm{p}=0.019$. The distribution of time played can be seen in Figure 8.
This change also improved player retention in Hello Worlds. 1007 players played Hello Worlds with on-path coins. When compared with the off-path coins condition, players with the on-path coins condition played significantly more levels, increasing from a median of 4 levels to a median of 7 levels, $\mathrm{Z}=-4.571, \mathrm{p}<0.0001$. Players with coins on the path also played significantly longer, increasing time played from a median of 360 seconds to 512 seconds, $\mathrm{Z}=-2.097, \mathrm{p}=$ 0.036. Finally, the return rate also increased from $18.37 \%$ to $23.24 \%, \chi^{2}=6.988, \mathrm{p}=0.008$. Compared with players in the no coins condition, the levels completed were not significantly different $(\mathrm{M}=7), \mathrm{Z}=-0.852, \mathrm{p}=0.394$. Players played longer with on-path coins $(\mathrm{M}=512)$ than with no coins $(\mathrm{M}=480)$, but not with statistical significance, $\mathrm{Z}$ $=-1.606, \mathrm{p}=0.108$. Players returned more with on-path coins, although not significantly: $23.24 \%$ of players with onpath coins returned, compared to $20.44 \%$ with no coins, $\chi^{2}=2.307, \mathrm{p}=0.129$. As seen in Figure 7, players who played Refraction with on-path coins played for longer than players with no coins or off-path coins. This can be seen in the populations which played for a moderate amount of time and those which played for a long time. Figure 8 shows the distribution of time played for Hello Worlds. As was the case in Refraction, players who played for a moderate amount of time with on-path coins played longer than players with offpath coins or no coins. In addition, the players who played for a long time played nearly as long as those with off-path coins.

These results show that we were able to reduce the negative effects of off-path coins by making the coins easier to obtain. In addition, we were able to keep most of the positive effects of off-path coins on players who played for a long time with on-path coins. This suggests that secondary objectives that support the primary objectives of the game increase player retention for both play time and levels played. Further re- 


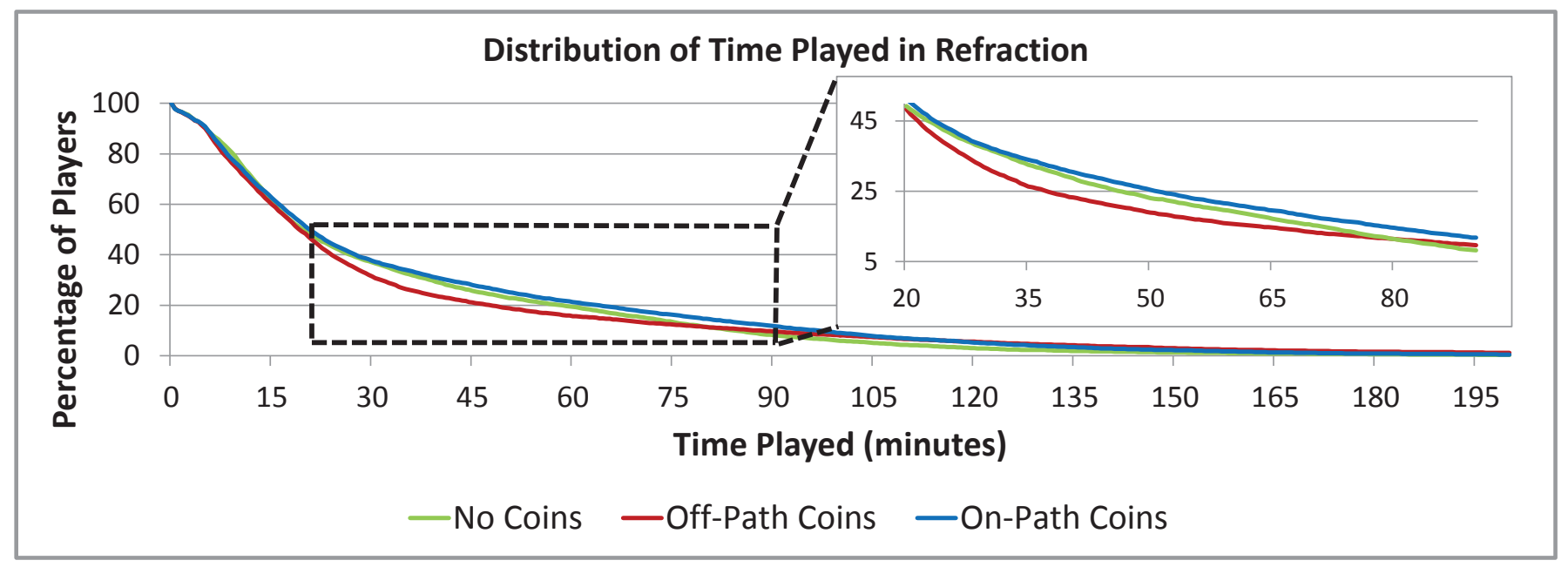

Figure 7: Graph of time played for Refraction, comparing the versions of the game with on-path, off-path, and no coins. On-path coins are those placed on the path to the solution, and are much easier to obtain compared to those placed off the path to the solution. On-path coins are superior in terms of player retention, avoiding the disadvantages that are present in the off-path and no coins conditions.

search is necessary to see if this result generalizes to other games and types of secondary objectives.

\section{CONCLUSION}

Our examination of two online Flash games shows that secondary objectives can negatively impact player retention if they are too challenging. Off-path coins distracted players from the primary goals of the game, causing them to complete fewer levels than players with no secondary objectives. In terms of overall play time, players reacted differently to difficult secondary objectives. Players who played for a moderate length of time quit earlier, while expert players who played for a long time played longer, presumably to go after the coins. In both games, the proportion of players who quit earlier due to off-path coins was surprisingly large; in Refraction, this group was four times as large as the group of players who played longer. Therefore, game designers should not assume that players will ignore secondary objectives if they find them too challenging, and should consider the possible harmful effects of player frustration.

By creating secondary objectives that support the primary objectives rather than distract from them, we were able to reverse these negative trends. Players with on-path coins who played for a moderate amount of time played longer than both players with no coins and players with off-path coins. Additionally, long-term players played for a longer time with on-path coins than players with no coins. As a result, the benefits of secondary objectives appear strongest when they directly support the main mission of the game because they increase the retention of long-term players without negatively impacting the rest of the population. While more work is necessary to know exactly what players will and will not tolerate, and how to find the optimal level of difficulty for secondary objectives, we find it encouraging that the same approach succeeded in both games.

Our results show that secondary objectives can have complex effects on player behavior, and that game designers should exercise caution to avoid unexpected negative consequences. A key question that arises is how these results generalize to other games and genres. There are many kinds of secondary objectives, and we only experimented with basic collectibles in a platformer game and side challenges in a puzzle game. Further experiments with a greater variety of secondary objectives and achievements are necessary to know how they impact player behavior as a whole. However, if the effects that we observed are present in many kinds of games and with many kinds of optional challenges, these results could have a profound effect on our understanding of player behavior and motivation.

Our findings also point to the importance of player metrics and large-scale measurement of player behavior because these effects are likely hard to detect with small amounts of player data. Many game designers and experts have strongly held beliefs about player motivation, but more numerical data is needed to support these recommendations. We believe that experimentation within games will help researchers evaluate the validity of these beliefs and discover general principles of player behavior and motivation. The venues for rapid and inexpensive distribution of games that have recently emerged, such as Kongregate, make this kind of iterative experimentation possible on a large scale.

\section{ACKNOWLEDGMENTS}

We thank Kongregate and the additional creators of Refraction and Hello Worlds: Ethan Apter, Brian Britigan, Eric Butler, Michael Eng, Mai Dang, Happy Dong, Jeff Flatten, Justin Irwen, Christian Lee, Marianne Lee, Emma Lynch, Stephen Sievers, and Blake Thompson. This work was supported by the University of Washington Center for Game Science, DARPA grant FA8750-11-2-0102, NSF grant IIS0811902, an NSF Graduate Fellowship, Adobe, Intel, and Microsoft. 


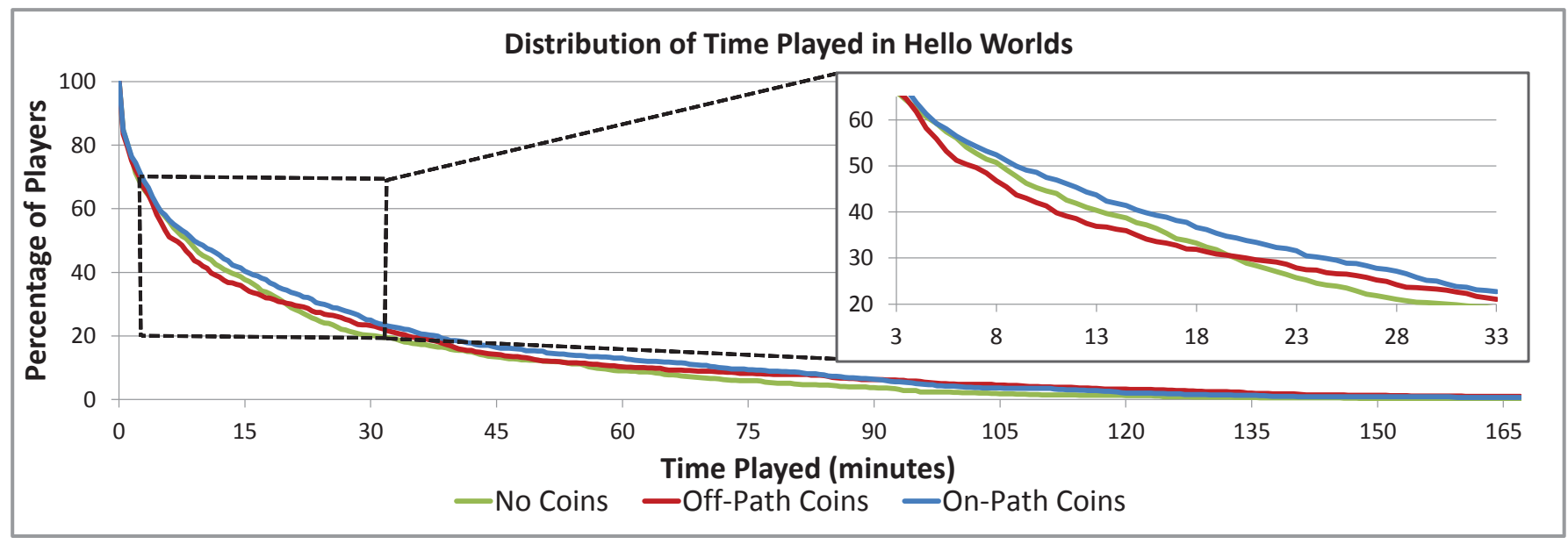

Figure 8: Graph of time played for Hello Worlds, comparing the versions of the game with on-path, off-path, and no coins. As in Refraction, the on-path coins improve player retention, also avoiding the disadvantages from the off-path and no coins conditions.

\section{REFERENCES}

[1] L. Alexander. Gamestop buys social gaming hub kongregate. Gamasutra, 2010.

[2] E. Andersen, Y.-E. Liu, R. Snider, R. Szeto, and Z. Popović. Placing a value on aesthetics in online casual games. In CHI '11: Proceedings of the SIGCHI conference on Human factors in computing systems, New York, NY, USA, 2011. ACM.

[3] R. Bartle. Hearts, clubs, diamonds, spades: players who suit MUDs. 1996. http://www.mud.co.uk/richard/hcds.htm.

[4] M. Csikszentmihalyi. Flow: The Psychology of Optimal Experience. Harper \& Row Publishers, Inc., New York, NY, USA, 1990.

[5] E. L. Deci and R. M. Ryan. Intrinsic Motivation. John Wiley \& Sons, Inc., 2010.

[6] A. Gazzard. Unlocking the gameworld: The rewards of space and time in videogames. Game Studies, 11, 2011.

[7] J. P. Gee. Learning by design: Games as learning machines. Interactive Educational Multimedia, 8:15-23, 2004.

[8] Google Website Optimizer. http://www.google.com/websiteoptimizer/.

[9] K. Graft. Dice 2010: Zynga's reynolds on 'social' first and foremost. Gamasutra, 2010.

[10] J. M. Harackiewicz and A. J. Elliot. Achievement goals and intrinsic motivation. Journal of Personality and Social Psychology, 65(5):904 - 915, 1993.

[11] K. Hullett and J. Whitehead. Design patterns in fps levels. In Proceedings of the Fifth International Conference on the Foundations of Digital Games, FDG '10, pages 78-85, New York, NY, USA, 2010. ACM.

[12] J. Klein, Y. Moon, and R. W. Picard. This computer responds to user frustration: Theory, design, and results. Interacting with Computers, 14(2):119 - 140, 2002.

[13] R. Kohavi, R. M. Henne, and D. Sommerfield. Practical guide to controlled experiments on the web: listen to your customers not to the hippo. In $K D D$ ' 0 '\%
Proceedings of the 13th ACM SIGKDD international conference on Knowledge discovery and data mining, pages 959-967, New York, NY, USA, 2007. ACM.

[14] C. Lewis and N. Wardrip-Fruin. Mining game statistics from web services: a world of warcraft armory case study. In Proceedings of the Fifth International Conference on the Foundations of Digital Games, FDG' 10, pages 100-107, New York, NY, USA, 2010. ACM.

[15] D. Milam and M. S. El Nasr. Analysis of level design 'push \& pull' within 21 games. In Proceedings of the Fifth International Conference on the Foundations of Digital Games, FDG '10, pages 139-146, New York, NY, USA, 2010. ACM.

[16] Nintendo. Super Mario Brothers. Console Game, 1985.

[17] R. Ryan, C. Rigby, and A. Przybylski. The motivational pull of video games: A self-determination theory approach. Motivation and Emotion, 30:344-360, 2006. 10.1007/s11031-006-9051-8.

[18] R. M. Ryan and E. L. Deci. Self-determination theory and the facilitation of intrinsic motivation, social development, and well-being. The American psychologist, 55(1):68-78, January 2000.

[19] J. Schell. The Art of Game Design: A Book of Lenses. Morgan Kaufmann Publishers, Burlington, MA, 2008.

[20] P. R. Selvidge, B. S. Chaparro, and G. T. Bender. The world wide wait: effects of delays on user performance. International Journal of Industrial Ergonomics, 29(1):15 - 20, 2002.

[21] G. Smith, M. Cha, and J. Whitehead. A framework for analysis of $2 \mathrm{~d}$ platformer levels. In Proceedings of the 2008 ACM SIGGRAPH symposium on Video games, Sandbox '08, pages 75-80, New York, NY, USA, 2008. ACM.

[22] N. Yee. Motivations for play in online games. CyberPsychology \& Behavior, 9:772-775, 2006. 